\title{
Repair of a Divergent Elbow Dislocation with Distal Ulnar Fracture in a Dog Using TightRope and External Skeletal Fixation
}

\author{
Marcos Garcia ${ }^{1}$ Camille Bismuth ${ }^{2}$ Claire Deroy-Bordenave ${ }^{3}$ \\ ${ }^{1}$ Department of Surgery, Centre Hospitalier Vétérinaire Saint-Martin, \\ Address for correspondence Marcos Garcia, Ldo, Med, Vet, Centre \\ Saint-Martin-Bellevue, France \\ Hospitalier Vétérinaire Saint-Martin (CHVSM), 275 Route Impériale, \\ 2 Departement of Surgery, Centre Hospitalier Vétérinaire Fregis,
} Arcueil, France

${ }^{3}$ Vet and Surg, Le Haillan, France

VCOT Open 2021;4:e58-e64.

\begin{abstract}
The aim of this study was to report the outcome in a 6-year-old male English Setter dog that suffered a combination of divergent elbow dislocation and open distal ulnar fracture. This study is a case report. After surgical reduction in the elbow luxation, the dog was treated with the TightRope fixation system used as replacement of the lateral

Keywords

- dog

- elbow luxation

- divergent dislocation

- TightRope

- Monteggia injury collateral ligament, a radioulnar positional screw, and external skeletal fixation. Removal of all implants was performed 3 months postoperatively. The 6-month follow-up visit found the dog without lameness, displaying normal activity, and with normal elbow range of motion and normal Campbell's test. Successful surgical management was achieved with good long-term results using TightRope, a positional screw, and an external skeletal fixator.
\end{abstract}

\section{Introduction}

Divergent elbow dislocation is defined as an elbow luxation in which the distal humerus is forced between the proximal radius and ulna, resulting in separation or divergence of the proximal antebrachial bones. ${ }^{1}$ The elbow joint can be divided into three synovial joints: humeroradial, humeroulnar, and proximal radioulnar. The humeroradial joint transmits most of the weight-bearing forces of the limb, the humeroulnar joint restricts the elbow joint's movement to the sagittal plane, whereas the radioulnar joint enables rotational, pronation, and supination movements. The joint capsule is shared by all three articular parts and the stability is conferred by periarticular and ligamentous structures. ${ }^{2,3}$ The primary stability of the elbow joint is provided by the collateral ligaments. ${ }^{4}$ Thus, although the joint is not protected by heavy muscles, severe rotational stress or direct force generally results in bone fracture rather than dislocation. ${ }^{2,5}$ The most

received

September 10, 2020

accepted after revision

April 24, 2021 frequent causes of luxation are road traffic accidents, falls, fights, and limb entrapments. ${ }^{5-7}$ Some authors claim that when traumatic dislocation occurs, soft tissue and ligament disruption is invariably associated. ${ }^{8,9}$ Others report ligament rupture in up to $50 \%$ of dislocations. ${ }^{5,7,9,10} \mathrm{~A}$ biomechanical cadaver study showed that luxation could not be generated by applying rotational forces with intact collateral ligaments. ${ }^{11} \mathrm{In}$ dogs, the direction of luxation is most common laterally because the medial epicondylar ridge of the humerus is larger than the lateral part and its articular surface is distally sloping. ${ }^{7,8}$

The divergent dislocation of the elbow is a rare combination of injuries to the elbow with only a few cases described in human medicine especially in children.1,12 To the authors' knowledge, this configuration of divergent elbow joint luxation associated with distal ulnar fracture has not previously been described in the dog. This report's aim is to describe the combined use of the TightRope (Arthrex Vet

\section{(c) 2021. The Author(s).}

This is an open access article published by Thieme under the terms of the Creative Commons Attribution License, permitting unrestricted use, distribution, and reproduction so long as the original work is properly cited. (https://creativecommons.org/licenses/by/4.0/) Georg Thieme Verlag KG, Rüdigerstraße 14, 70469 Stuttgart, Germany 
Systems, Creekside Boulevard, Naples, Florida, United States) system and external skeletal fixation in a client-owned dog with divergent elbow dislocation and report short- and longterm outcomes.

\section{Case Description}

A 6-year-old male English Setter of $26 \mathrm{~kg}$ was referred with non-weight-bearing lameness of the left forelimb after having been bitten by another dog 12 hours earlier. Referring veterinarian has administrated methadone $(0.2 \mathrm{mg} / \mathrm{kg}$ intravenously [IV]) and cephazolin ( $20 \mathrm{mg} / \mathrm{kg}$ IV) at the emergency consultation. On presentation, the left forelimb was unsupported, without weight-bearing, and with the elbow joint fixed in slight flexion. Examination revealed severe decrease in the left elbow's range of motion, crepitus, pain, and considerable swelling of the antebrachium. Swelling in the distal antebrachium, pain, and crepitus in the distal ulna with mild lateral antebrachiocarpal instability of the left carpus were detected. A 7-mm clean wound was observed on the lateral distal third of the antebrachium. There were no apparent neurological deficits.

Thorax radiographs, haematology, and serum biochemistry profiles were unremarkable.

Orthogonal radiographs of the left forelimb were obtained, revealing radioulnar luxation with craniolateral displacement of the radial head and humeroulnar luxation with lateral rotation of the proximal part of the ulna. There was a divergent dislocation of the elbow (-Fig. 1A and B). The radiographs also showed a displaced distal short oblique ulnar fracture.

The next day the patient was anaesthetized. After premedication with diazepam (Solupam, Dechra Veterinary Products SAS, Montigny-le-Bretonneux, France; $0.2 \mathrm{mg} / \mathrm{kg}$ IV) and methadone (Comfortan, Dechra Veterinary Products SAS, Montigny-le-Bretonneux, France; $0.2 \mathrm{mg} / \mathrm{kg}$ IV), general anaesthesia was induced with propofol (Propovet, Zoetis France SAS, Malakoff, France; $1 \mathrm{mg} / \mathrm{kg}$ IV), followed by isoflurane (Isoflu-vet, Dechra Veterinary Products SAS, Montigny-le-Bretonneux, France) and $100 \% \mathrm{O}_{2}$. Lactated Ringer's solution (Soluté injectable de Ringer lactate Ecoflac, Virbac France, Carros Cedex, France; $5 \mathrm{~mL} / \mathrm{kg} / \mathrm{h}$ IV) was administered intraoperatively. Antibiotic prophylaxis was ensured by cephazolin (Cefazoline Mylan $1 \mathrm{~g}$, Mylan S. Saint-Priest, France; $22 \mathrm{mg} / \mathrm{kg}$ IV, repeated q.90 minute).

Surgery was performed aiming to reduce and stabilize the elbow luxation and distal ulnar fracture. The dog was placed in right lateral recumbency for a caudolateral approach to the elbow with separation of the anconaeus muscle. A complete inspection of the elbow joint revealed disruption of the annular and the cranial crura of the lateral collateral ligaments. The radial head was luxated craniolaterally and the radius was displaced proximally ( $\mathbf{- F i g}$. 2A). The proximal ulna was found rotated 60 degrees and luxated laterally (-Fig. 2B).

Reduction in the humeroulnar joint was accomplished by internal rotation of the proximal ulna with a point-to-point reduction forceps placed at the olecranon and by pressure applied to its lateral surface. The reduction in the radial head was then performed by applying steady lateral-to-medial pressure to the head of the radius over several minutes to allow the soft tissues to stretch. Reduction in the radial head was very difficult to achieve; therefore, it was performed by manual pressure with a point-to-point reduction forceps from the radial head to the ulna and a Freer periosteal elevator held between the radial head and the humeral condyle to level the radius. Once the elbow luxation was reduced, the integrity of the joint was assessed by means of Campbell's test revealing the laterolateral instability of the joint. ${ }^{5}$ Further manipulation of the elbow caused it to reluxate, in particular the radial head. Reduction in the radial head and the proximal ulna was reproducible. As the elbow
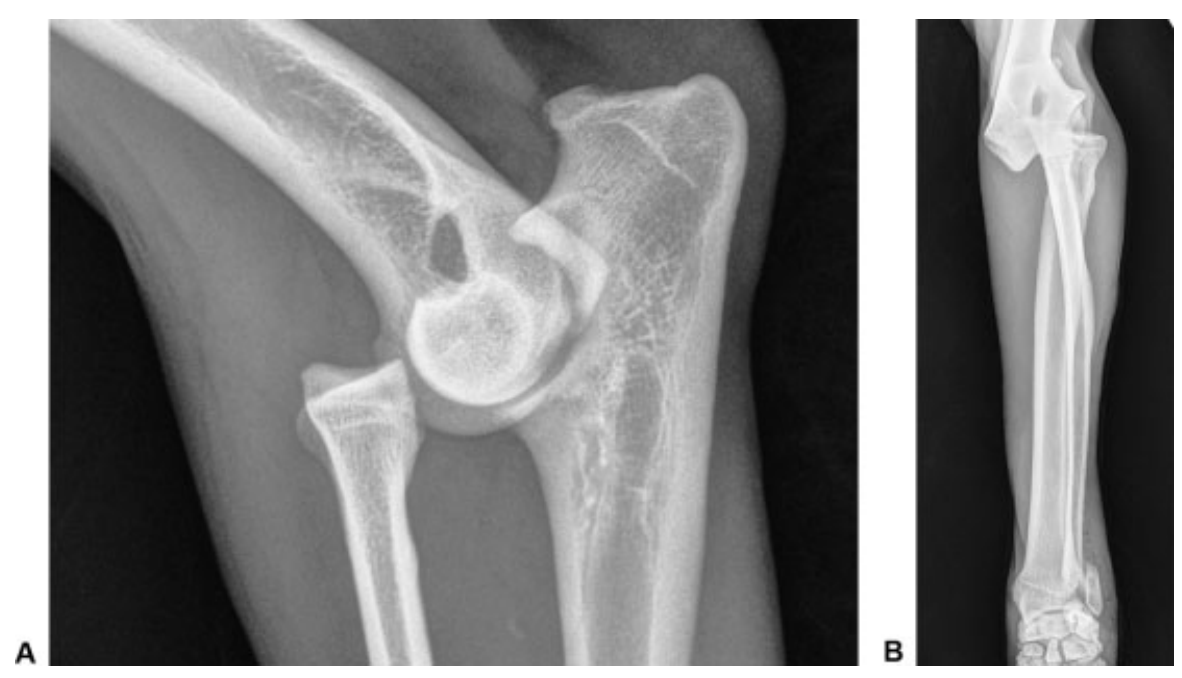

Fig. 1 Orthogonal radiographs of the left forelimb revealing a divergent luxation of the elbow. (A) Mediolateral radiograph of the left elbow showing cranial displacement of the radial head. (B) Craniocaudal radiograph of the left antebrachium revealing lateral displacement of the radial head and humeroulnar luxation with lateral rotation of the proximal part of the ulna, and a displaced distal short oblique ulnar fracture with humeroradioulnar luxation. 

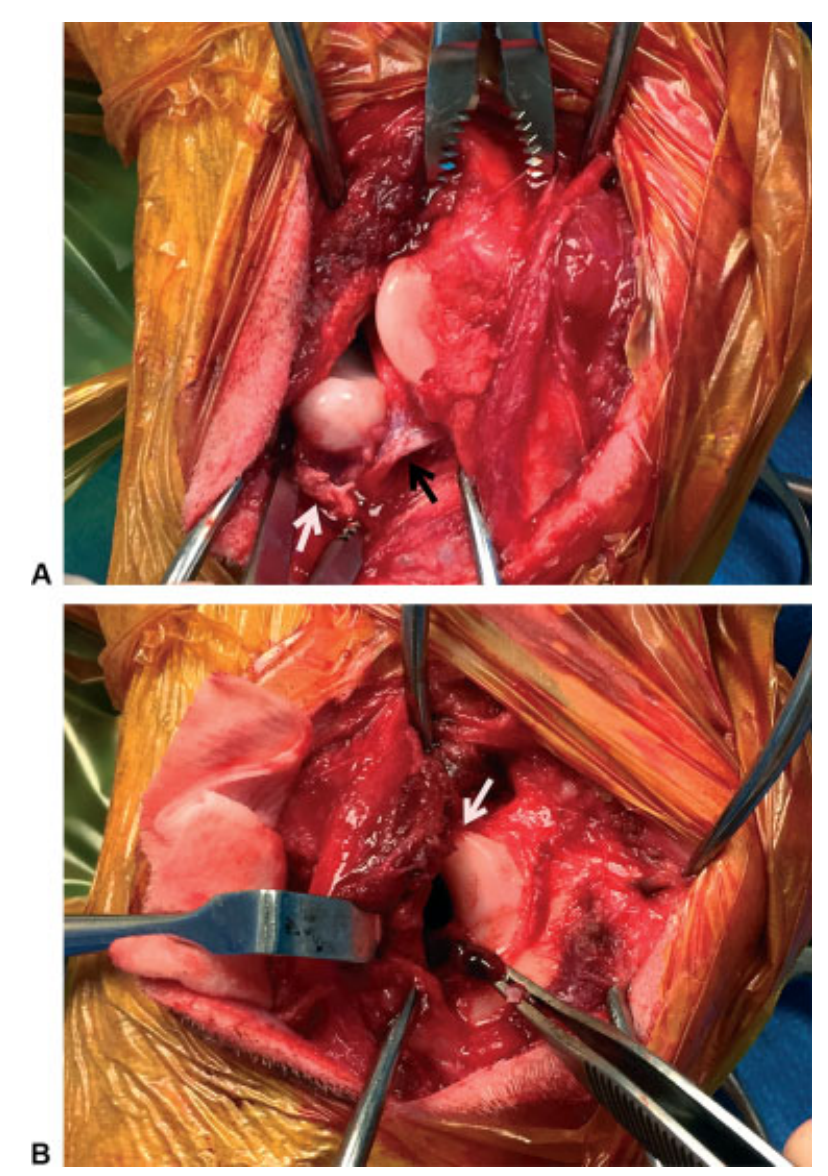

Fig. 2 Intraoperative medial view of the elbow. (A) Craniolateral luxation of the radial head with proximal displacement. The annular ligament and the cranial crura of the lateral collateral ligament appear disrupted, indicated, respectively, by a white arrow and a black arrow. (B) Proximal ulna rotated 60 degrees laterally and luxated laterally. The anconeal process was indicated by a white narrow.

was unstable after reduction, surgical stabilization of the luxated elbow was indicated.

With the radial head held in reduction, a positional cortical screw (ø $2.7 \mathrm{~mm}$ ) was inserted through the caudal surface of the ulna to project through the cranial cortex of the radius, so as to hold the radius and ulna in apposition.

A standard-size TightRope comprised of a $1.6-\mathrm{mm}$ guide pin, a 2-0 double-stranded FiberWire suture, a 3-mm alloy toggle and a $7.5 \times 12-\mathrm{mm}$ surgical button was placed. A guide wire for a cannulated drill bit (Cannulated Drill Bit, Arthrex Vet Systems, Creekside Boulevard, Naples, Florida, United States) was used to drill three bone tunnels from lateral to medial with a $1.6 \mathrm{~mm}$ guide pin: humeral transcondylar, transradial, and transulnar. The humeral transcondylar tunnel was made at the origin of the lateral collateral ligament immediately craniodistal to the lateral epicondyle and directed toward a point craniodistal to the medial epicondyle. The transradial tunnel was made at the insertion of the lateral collateral ligament on the radial head, whereas the transulnar tunnel was made in the proximal ulna at the level of the mid-portion of the ulnar trochlear notch midway between the articular surface and the caudal ulnar cortex.

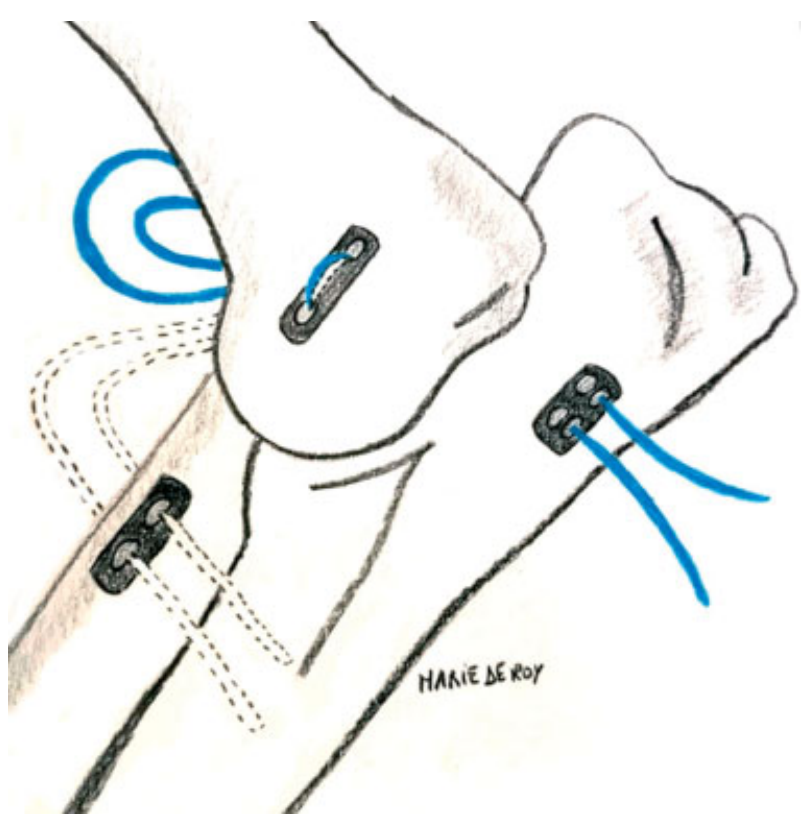

Fig. 3 Positioning of the transcondylar, transradial and transulnar tunnels with TightRope system placement.

The wires were over-drilled with a $3.5-\mathrm{mm}$ cannulated drill bit and a standard-size TightRope with 4 strands was placed. The swaged TightRope needle with the toggle button was inserted through the transradial tunnel in a medial-tolateral direction, then through the humeral transcondylar tunnel in a lateral-to-medial direction. Once the toggle button had exited the humeral transcondylar tunnel medially, the toggle button was flipped to be seated on the medial humeral condyle by pulling back on the TightRope suture strands laterally. The two strands removed from the transradial tunnel were placed in the transulnar tunnel from lateral to medial. Those two strands were inserted in a button. The toggle button and the button were compressed against the medial cortex of the radius and ulna respectively (-Fig. 3). The elbow joint as assessed by the Campbell's test was stable with TightRope replacing the lateral collateral ligament. Thus, the cranial crura thereof were not reinforced and the TightRope was secured in position by five throws resulting in a stable elbow joint.

The ulnar fracture was approached caudolaterally over the styloid ulnar process and was reduced, and two $1.5-\mathrm{mm}$ pins were placed in the styloid ulnar process to the radius.

A type IA external fixator was installed with two threaded pins in the proximal ulna and two threaded pins in the distal radius to protect the radioulnar screw.

Postoperative radiographs revealed appropriate alignment and positioning of the implants except for the radial toggle position that was not in contact with the bone. Radiographs confirmed anatomic reduction in the radial head, humeroulnar joint, and ulnar fracture (-Fig. 4).

\section{Postoperative Care and Follow-Up}

Analgesia was provided with methadone for the first 24 hours, followed by tramadol (Tralieve, Dechra Veterinary Products SAS, Montigny-le-Bretonneux, France; $5 \mathrm{mg} / \mathrm{kg}$ per 

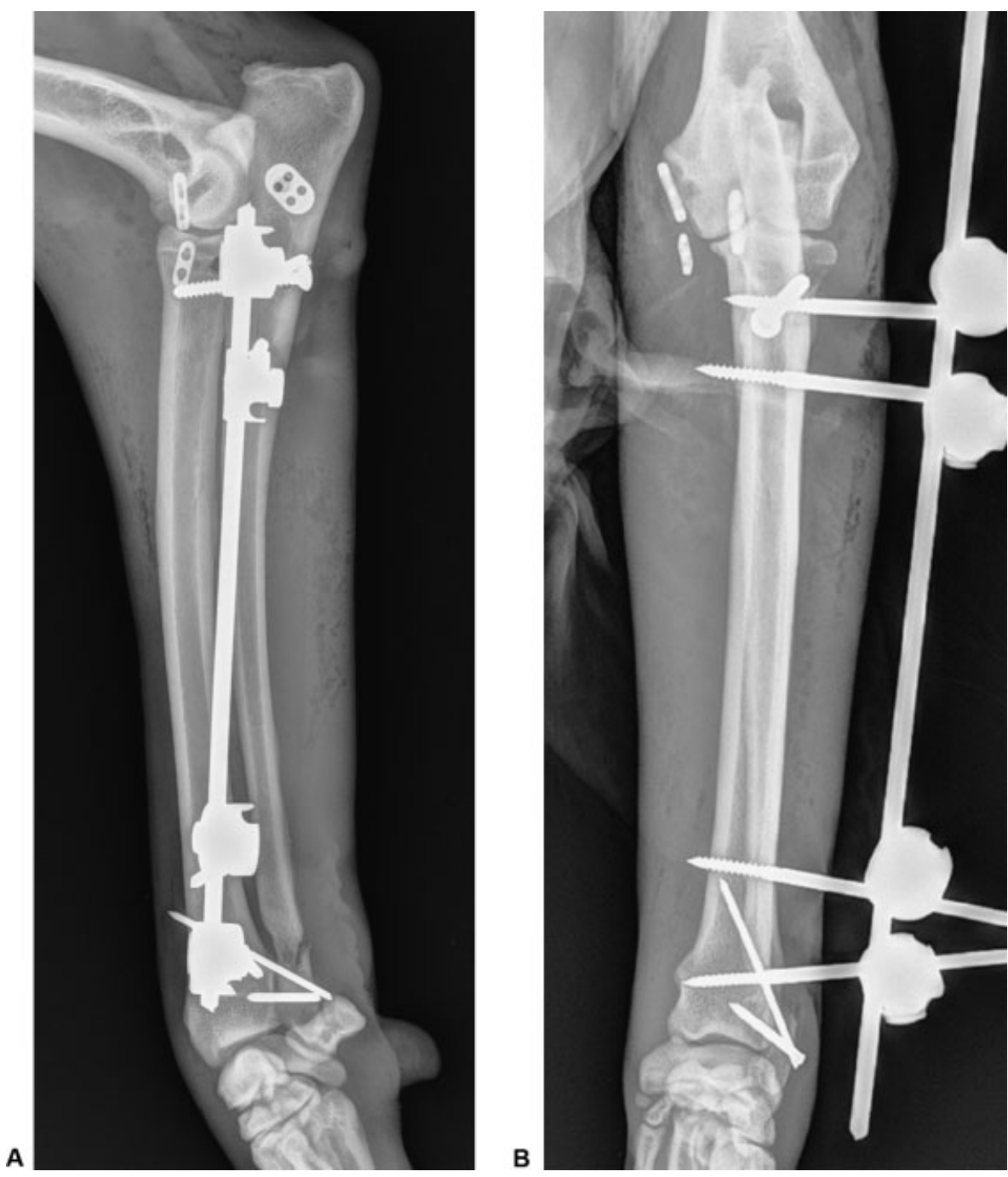

Fig. 4 Immediate postoperative radiographs showing elbow luxation reduction. The TightRope system, a radioulnar positional screw, two pins in the styloid process and an external skeletal fixator are seen. (A) Mediolateral radiograph of the left antebrachium. (B) Craniocaudal radiograph of the left antebrachium.

per os [PO] q. 8-12h). Cephazolin (Cefazoline Mylan, Mylan SAS, Saint-Priest, France) administration (22 mg/kg IV q.12h) was continued until discharge. The dog was discharged 2 days postoperatively with instructions to administer meloxicam (Metacam, Boehringer Ingelhheim, Reims CEDEX, France; $0.1 \mathrm{mg} / \mathrm{kg}$ PO q. $24 \mathrm{~h}$ for 10 days), tramadol ( $5 \mathrm{mg} / \mathrm{kg}$ PO q.12h for 7 days), and cephazolin (Therios, CEVA Santé Animale, Libourne, France; $20 \mathrm{mg} / \mathrm{kg}$ PO q. $12 \mathrm{~h}$ for 14 days). The dog's activity was restricted for 8 weeks.

Orthopaedic examination 4 weeks after surgery revealed a mild, weight-bearing lameness and moderate muscle atrophy of the left forelimb. Radiographs showed stable implants, normal bone remodelling at the distal ulna, and anatomic reduction in the elbow luxation. Palpation of the elbow joint exhibited normal extension and mildly reduced flexion.

\section{Outcome}

Orthopaedic examination 3 months after surgery showed a moderate weight-bearing lameness associated with decreased range of motion of the left elbow and carpus, and pain when manipulating the elbow. Also, there was a purulent discharge at the proximal pin of the external fixator. The external fixator was removed resulting in improved lameness a few days later. Physiotherapy exercises were recommended to increase range of motion in the elbow and carpus.

Radiographs at the 3-month follow-up visit revealed osteolysis at the positional screw head. The elbow was stable with improved range of motion, but a mild weight-bearing lameness persisted, and pain was elicited by applying pressure over the screw. All implants were removed, and their culture grew Staphylococcus intermedius, which was sensitive to broadspectrum antibiotic medications. Amoxicillin/clavulanate (Kesium, Ceva Santé Animale, Libourne, France) was prescribed $(15 \mathrm{mg} / \mathrm{kg}$ PO q. $12 \mathrm{~h}$ for 14 days).

Re-examination conducted 6 months postoperatively revealed return to normal activity. The elbow's range of motion and supination-pronation motion of the antebrachium were similar when clinically compared with their normal contralateral counterpart. Bilateral muscle symmetry was present.

Radiographs at the 16 months postoperatively showed union of the ulnar fracture, elbow congruence, and mild 

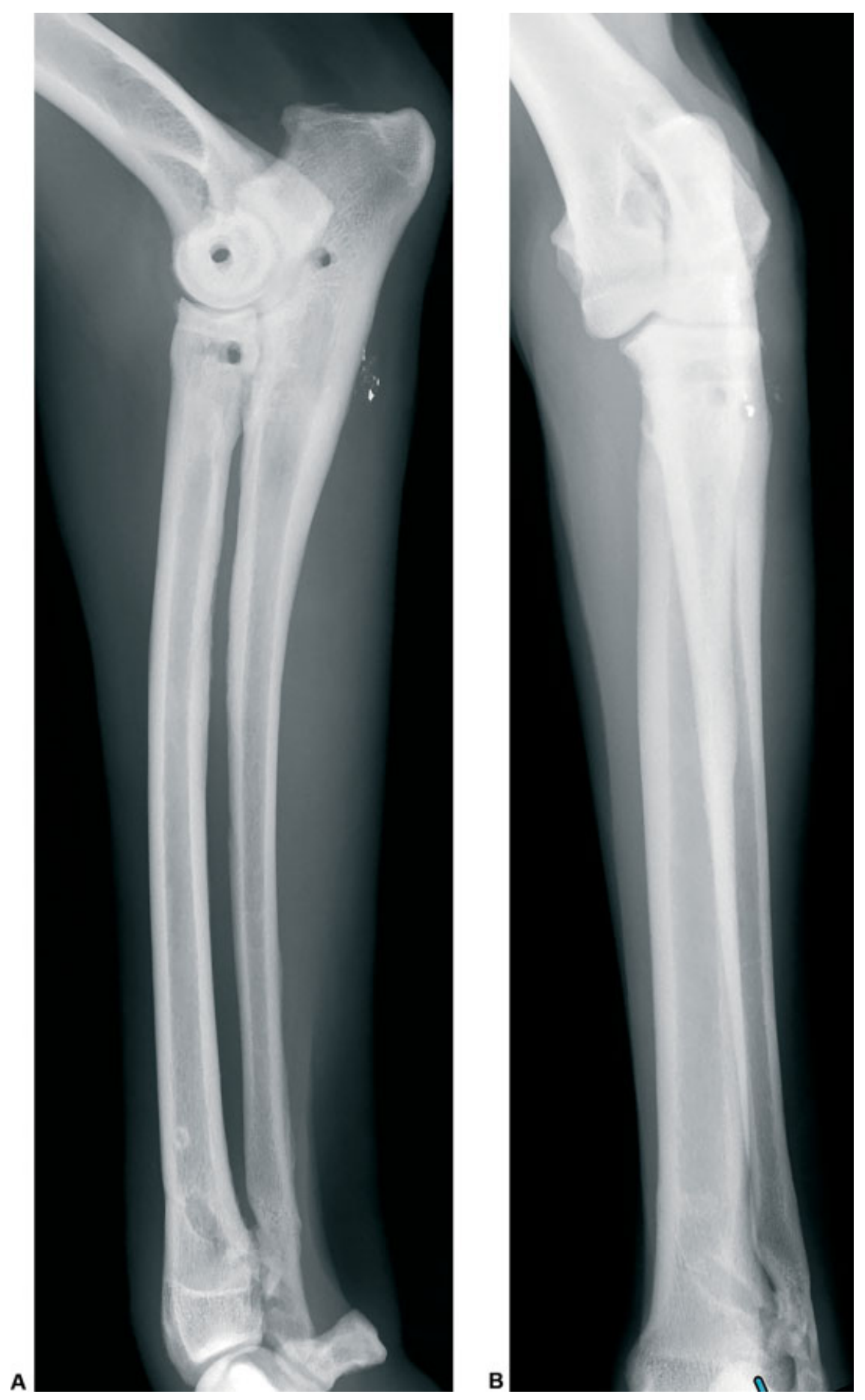

Fig. 5 Sixteen months postoperative mediolateral (A) and craniocaudal (B) antebrachial radiographs showing radiographic union of the ulnar fracture, elbow congruence and mild osteoarthritis of elbow joint.

osteoarthritis of the elbow joint (-Fig. 5). Long-term management of osteoarthritis, including a therapeutic diet formulated for joint health, and dietary supplementation were advised.

\section{Discussion}

We report the successful repair of a divergent elbow luxation and ulnar fracture caused by a dog bite, achieved by intraoperative manual reduction and stabilization using Tight-
Rope, a positional screw, and an external skeletal fixator. The dog returned to normal function with excellent range of motion 6 months after the procedure.

The term "divergent elbow dislocation" is based on the position of the proximal radius and ulna when both are luxated. This injury involves disruption of the proximal radioulnar joint and, consequently, the radius and the ulna displace in opposite directions away from each other. ${ }^{12}$ This terminology has not been previously used in veterinary medicine. This injury usually involves axial compressive 
and rotational forces on the humerus frequently incurred after a fall on an extended and pronated limb. ${ }^{13}$ Lateral luxation of the elbow has been hypothesized to occur as a result of direct force on the joint or the indirect application of rotational forces transmitted to the elbow via bridging ligaments and regional musculature. ${ }^{7,14}$ In the present dog, the injury could have been caused by a rotational force on an extended and pronated member exerted by the bite on the distal part of the limb.

In 1967, Bado described the Monteggia lesion as a dislocation of the radiohumeroulnar joint and a fracture of the ulna at any level. ${ }^{15}$ Pursuant to this definition, the orthopaedic injury in our case may be categorized as a Monteggia injury. The presence of a radiohumeral luxation, as with all Monteggia lesions, may suggest disruption of the joint capsule and the medial and lateral collateral ligaments. The ulna usually fractures distally to the coronoid process; however, the site of fracture may vary from the trochlear notch to the distal ulnar diaphysis. Ulnar fractures distal to the coronoid process necessarily disrupt the annular ligament and at least the proximal portion of the interosseous membrane and ligaments. Nevertheless, in other Monteggia fractures, such as in type II, the interosseus ligament and interosseus membrane may remain preserved. ${ }^{16,17}$

In this case, the cranial crura of the lateral collateral ligament and the annular ligament were damaged. The interosseous ligament, although not visualized during surgery, was also probably damaged considering the distance between radius and ulna observed on preoperative radiographs. When the annular ligament is torn, a positional screw across the radius and ulna immediately adjacent to the elbow joint may be placed to hold the radius and ulna in apposition, as herein presented.

The TightRope system has been used previously in Monteggia fracture repair to functionally replace the annular ligament, resulting in excellent clinical results. ${ }^{17}$ Also, a type II Monteggia fracture with concurrent humeroulnar luxation in a cat and its repair using a locking compression plate and circumferential sutures was reported recently. ${ }^{18}$ The TightRope system was used in the present case to replace the two crura of the lateral collateral ligament. The procedure was easy and quick to perform.

The instability of the carpus was associated with the distal ulna fracture in relation with the proximal attachment of the respective lateral collateral ligament. ${ }^{2} \mathrm{~A}$ simple repair using two pins was achieved. Given the dog's energetic character, the surgical repair was protected by the use of external skeletal fixation. Bandage was not employed due to the presence of an open fracture.

Bacterial culture and sensitivity testing were not performed at the time of surgery due to prior administration of antibiotic medications.

Notwithstanding the use of strict aseptic technique and remote location of the bite wound from the surgical site, infection developed. Since FiberWire is a braided suture, aseptic technique should be strictly adhered to prevent infection. Removal of all implants enabled infection resolution.
The most common complication after Monteggia lesion repair is osteoarthritis with consequent long-term elbow range of motion reduction. ${ }^{15,16}$ In the present case, a mild osteoarthritis was observed without evidence of clinical signs.

So far, TightRope applications in veterinary medicine included treatment of cranial cruciate ligament rupture, stabilization of stifle joint luxation or hip stabilization as a modified hip toggle. ${ }^{19-21}$

In conclusion, in the described case, using the TightRope system was successful in the management of this rare form of elbow joint luxation: 'the divergent dislocation of the elbow'; wider use of this terminology, hitherto known only in human medicine, would enable to better describe these types of lesions in our domain and could help improve surgical planning. Additional research of the pathophysiology, prevalence of such injuries in small animals, and further developing the described repair technique should be considered.

\section{Authors Contributions}

Claire Deroy-Bordenave performed the surgery and revised the manuscript.

Marcos Garcia assisted on the surgery, wrote, and submitted the manuscript.

Camile Bismuth participated in the corrections.

\section{Funding}

None.

\section{Conflict of Interest}

None declared.

\section{Acknowledgments}

The authors would like to thank referring veterinarian (Nicolas Perin) for his contribution to the postoperative radiographs and Marie Deroy for the diagram creation.

\section{References}

1 Altuntas AO, Balakumar J, Howells RJ, Graham HK. Posterior divergent dislocation of the elbow in children and adolescents: a report of three cases and review of the literature. J Pediatr Orthop 2005;25(03):317-321

2 Evans E, de Lahunta A. Miller's Anatomy of the Dog. 5th edition Missouri: Elsevier Saunders; 2019:169-172

3 Knox VW IV, Sehgal CM, Wood AKW. Correlation of ultrasonographic observations with anatomic features and radiography of the elbow joint in dogs. Am J Vet Res 2003;64(06):721-726

4 Talcott KW, Schulz KS, Kass PH, Mason DR, Stover SM. In vitro biomechanical study of rotational stabilizers of the canine elbow joint. Am J Vet Res 2002;63(11):1520-1526

5 Campbell JR. Luxation and ligamentous injuries of the elbow of the dog. Vet Clin North Am 1971;1(03):429-440

6 Sajik D, Meeson RL, Kulendra N, et al. Multi-centre retrospective study of long-term outcomes following traumatic elbow luxation in 37 dogs. J Small Anim Pract 2016;57(08):422-428

7 O'Brien MG, Boudrieau RJ, Clark GN. Traumatic luxation of the cubital joint (elbow) in dogs: 44 cases (1978-1988). J Am Vet Med Assoc 1992;201(11):1760-1765

8 Billings LA, Vasseur PB, Todoroff RJ, et al. Clinical results after reduction of traumatic elbow luxation in nine dogs and one cat. J Am Anim Hosp Assoc 1992;28:137-142 
9 Schaeffer IGF, Wolvekamp P, Meij BP, et al. Traumatic luxation of the elbow in 31 dogs. Vet Comp Orthop Traumatol 1999;12:33-39

10 Campbell JR. Nonfracture injuries to the canine elbow. J Am Vet Med Assoc 1969;155(05):735-744

11 Farrell M, Draffan D, Gemmill T, Mellor D, Carmichael S. In vitro validation of a technique for assessment of canine and feline elbow joint collateral ligament integrity and description of a new method for collateral ligament prosthetic replacement. Vet Surg 2007;36(06):548-556

12 Parikh SN, Lykissas MG, Mehlman CT, et al. Convergent and divergent dislocation of the pediatric elbow: two case reports and comprehensive review of literature. J Pediatr Orthop B 2014; 23(02):158-167

13 Thompson GH. Dislocations of the elbow. In: Beaty JH, Kasser JR, eds. Rockwood and Wilkins' Fractures in Children. Philadelphia: Lippincott Williams \& Wilkins; 2002:705-739

14 Farrell M, Thomson DG, Carmichael S. Surgical management of traumatic elbow luxation in two cats using circumferential suture prostheses. Vet Comp Orthop Traumatol 2009;22(01): 66-69
15 Bado JL. The Monteggia lesion. Clin Orthop Relat Res 1967;50(50): 71-86

16 Schwarz PD, Schrader SC. Ulnar fracture and dislocation of the proximal radial epiphysis (Monteggia lesion) in the dog and cat: a review of 28 cases. J Am Vet Med Assoc 1984;185(02):190-194

17 Vallone L, Schulz K. Repair of Monteggia fractures using an Arthrex Tightrope system and ulnar plating. Vet Surg 2011;40(06):734-737

18 Fernandez-Sanchez A, Calvo I, Farrel M. Articular type II Monteggia fracture with concurrent humeroulnar luxation in a Bengal cat. Vet Rec Case Rep 2019;7:e000697

19 Kieves NR, Lotsikas PJ, Schulz KS, Canapp SO. Hip toggle stabilization using the TightRope ${ }^{\circledR}$ system in 17 dogs: technique and longterm outcome. Vet Surg 2014;43(05):515-522

20 Cook JL, Luther JK, Beetem J, Karnes J, Cook CR. Clinical comparison of a novel extracapsular stabilization procedure and tibial plateau leveling osteotomy for treatment of cranial cruciate ligament deficiency in dogs. Vet Surg 2010;39(03):315-323

21 Brand B, Lautersack O. Die Behandlung der Kniegelenksluxation der Katze mittels TightRope ${ }^{\circledR}$ [The treatment of stifle joint luxation in the cat with TightRope ${ }^{\circledR}$ ]. Kleintierpraxis 2012;57:305-312 